\title{
Comportamiento fisiológico y fisicoquímico de frutos de curuba (Passiflora mollissima Bailey) encerados y almacenados a dos temperaturas
}

\section{Physiological and physicochemical behavior of coated banana passion fruit (Passiflora mollissima Bailey) stored at two temperatures}

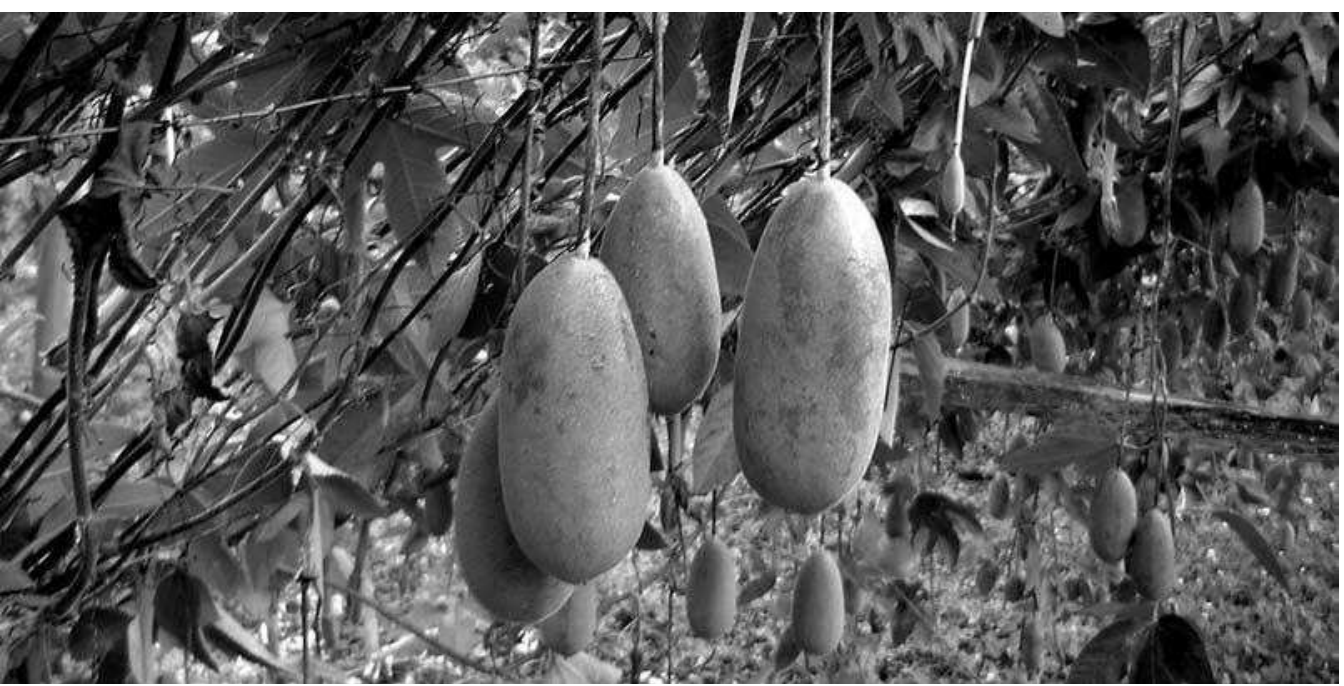

CLAUDIA PATRICIA TÉLLEZ

GERHARD FISCHER ${ }^{2}$

OMAR CAMILO QUINTERO 3

Cultivo de curuba en producción.

Foto: 0. C. Quintero

\section{RESUMEN}

Con el objeto de evaluar el comportamiento poscosecha de curubas, provenientes de la finca "Pozo Hondo" (vía Facatativá-Anolaima, Cundinamarca), los cultivares Ruizquin 1 (R1) y Ruizquin 2 (R2) fueron sometidos o no al tratamiento de encerado con la cera Primafresh (mezcla de ésteres ácido-grasos-sacarosa) y almacenados a $8^{\circ} \mathrm{C}(85 \% \mathrm{HR})$ o $20^{\circ} \mathrm{C}(53 \% \mathrm{HR})$, durante 25 y 55 días para frutos no encerados y encerados, respectivamente. Los frutos de todos los tratamientos mostraron un comportamiento climatérico. El pico respiratorio ocurrió más pronto en frutos sin cera, a $20^{\circ} \mathrm{C}$ entre los días 11 (R1) y 12 (R2) y a $8^{\circ} \mathrm{C}$ entre 12 (R2) y 14 (R1) días. Los frutos encerados tuvieron tasas respiratorias más bajas y un pico climatérico más tardío, a $20^{\circ} \mathrm{C}$ a los 31 (R1) y 30 días (R2) y a $8^{\circ} \mathrm{C}$ a los 31 (R1) y 38 días (R2); en este último tratamiento se registraron las tasas más bajas durante el pico climatérico $\left.\left[28,97 \mathrm{mg} \mathrm{CO} \cdot \mathrm{kg}^{-1} \cdot \mathrm{h}^{-1}\right]\right)$. Los frutos encerados tuvieron un $\mathrm{pH}$ y una acidez total titulable (ATT) mayor que los no encerados. El peso fresco del fruto y la consistencia de pulpa se conservaron más en fruta encerada, mientras el contenido de sólidos solubles totales (SST) y la relación SST/ATT fue mayor en frutos sin cera. El solo enfriamiento generó una pérdida más lenta de peso y consistencia, una intensidad

Ingeniera Agrícola, Facultad de Ingeniería, Universidad Nacional de Colombia, Bogotá. claudiapt@gmx.de Profesor Asociado, Facultad de Agronomía, Universidad Nacional de Colombia, Bogotá. gerfischer@gmail.com Biólogo, DisFruta las Feijoas, Bogotá.overfeijoas@gmail.com 
respiratoria más baja y un retardo del climaterio, una mayor cantidad de SST y ATT, un menor pH y una menor relación SST/ATT, comparado con la curuba a $20^{\circ} \mathrm{C}$.

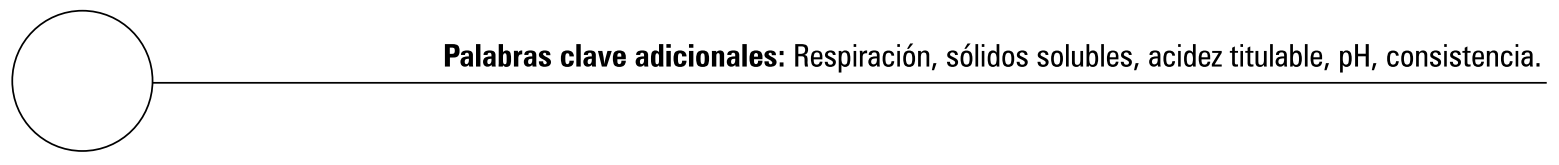

\section{ABSTRACT}

The objective of the study was to evaluate postharvest behavior of curuba fruits of cultivars Ruizquin CR1 and Ruizquin CR2 coated with Primafresh wax (mixture of acid-fatty-sucrose esters) and then stored at $8^{\circ} \mathrm{C}(85 \% \mathrm{RH})$ or $20^{\circ}(53 \% \mathrm{RH})$ during 25 and 55 days for fruits with or without coating, respectively. Fruits of all treatments had a climacteric behavior. The climacteric peak occurred faster in fruits without coating, between 11 (R1) and 12 days (R2) at $20^{\circ} \mathrm{C}$ and between $12(\mathrm{R} 2)$ and 14 days (R1) at $8^{\circ} \mathrm{C}$. Coated fruits had lower respiration rates and showed a climacteric peak later, at $20^{\circ} \mathrm{C}$ at 31 (R1) and 30 days (R2), at $8^{\circ} \mathrm{C}$ at 31 days (R1) and 38 days (R2); in the latest treatment the lowest rates during the climacteric peak were registered $\left(28,97 \mathrm{mg} \mathrm{CO} \cdot \mathrm{kg}^{-1} \cdot \mathrm{h}^{-1}\right)$. Coated fruits had a higher $\mathrm{pH}$ and total titratable acid content (TTA) than fruits without waxing. The fruit fresh weight and pulp firmness were better conserved in coated fruits, while total soluble solids (TSS) content and TSS/TTA relation were higher in uncoated fruits. Only cold storage of fruits resulted in a slower loss of weight and firmness, lower respiration rate and decay of climacteric point, increase in TSS and ATT, lower pH, and lower TSS/TTA ratio compared to curuba fruits stored at $20^{\circ} \mathrm{C}$.

Additional key words: Respiration, soluble solids, titratable acidity, pH, firmness.

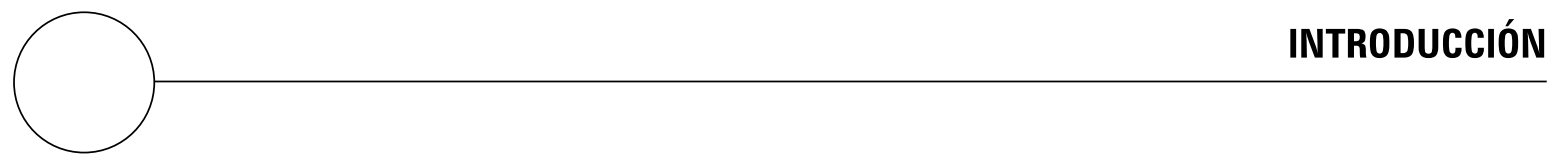

La curuba (Passiflora mollissima Bailey) es una especie originaria de la zona andina (Colombia, Ecuador, Perú y Bolivia). El nombre mollissima, que significa muy suave, muy blando, hace referencia a sus frutos blandos y al vello delicado que cubre todos los órganos vegetativos de la planta (Hoyos, 1989). En Colombia sus cultivos se localizan a lo largo de las tres cordilleras, en especial en la Oriental y Central, y con el correr de los años, específicamente a partir de los años cincuenta, se han venido estableciendo plantaciones comerciales (Campos, 1992).

Actualmente, en Colombia, según el Plan Frutícola Nacional, el área sembrada de curuba corresponde a unas 1.824 ha, destacándose los departamentos de Boyacá (1.122 ha) y Cundinamarca (110 ha) (Tafur, 2006); Angulo y Fischer (1999) reportan rendimientos de $8 \mathrm{t} \cdot \mathrm{ha}^{-1} \cdot \mathrm{a}^{-1}$. El cultivo de la curuba ofrece muchas expectativas debido a la 
gran aceptación de este producto en el mercado externo, la tendencia de consumir cada vez más frutas y los deseos en los países desarrollados por el consumo de exóticas, hacen de la curuba una fruta con grandes perspectivas en el mercado internacional (Campos, 2001).

Nakasone y Paull (1998) estiman la pérdida en poscosecha de las diferentes especies de frutas tropicales entre 10 y $80 \%$ y constatando que una reducción de esta cifra solamente se puede lograr con un amplio conocimiento de la fisiología en poscosecha y una aplicación de esto a una tecnología adecuada de almacenamiento. Kader (2002) afirma que la velocidad de deterioro de los productos hortofrutícolas después de cosechados (por ejemplo pérdida de peso y consistencia o maduración acelerado), está asociada con el manejo que recibe y con las condiciones de almacenamiento, transporte y mercadeo.

En muchas frutas y hortalizas frescas se están aplicando diferentes ceras para incrementar la resistencia de la piel a la difusión de gases, modificar la composición de su atmósfera interna, deprimir su tasa de respiración y reducir las pérdidas de agua por transpiración (Banks et al., 1993), con el fin de aumentar la vida útil en la poscosecha. Además de estos procesos fisiológicos, Fonade (1988) destaca otras ventajas del encerado, como: (a) formar una barrera física adicional contra ataques de microorganismos y mohos, (b) sellar los rasguños o cortes que se producen en la epidermis de la frutas, (c) reducir el daño ocasionado por el roce entre las frutas, (d) cumplir con los requerimientos de brillo de los mercados domésticos y de exportación y, (e) secar rápidamente, lo que permite procesar altos volúmenes de fruta.

Algunos frutos se enceran para remplazar la cera natural después de haberla eliminado durante el proceso de lavado y, además, puede actuar como un "transportador" (Carrier) para fungicidas; en piña reporta Kader (2002) que el encerado del fruto estimula la acumulación de $\mathrm{CO}_{2}$ hasta 5-
$8 \%$ como tratamiento efectivo en la reducción de síntomas de daño por frío.

Según Baldwin et al. (1995), la cobertura ideal comestible del fruto debe crear una barrera, la cual puede retardar las pérdidas de sustancias volátiles deseadas del sabor y, las del vapor de agua, mientras restringe el intercambio del $\mathrm{CO}_{2}$ $\mathrm{y}_{2}$, originando, así, una atmósfera modificada (con el $\mathrm{CO}_{2}$ relativamente elevado y el $\mathrm{O}_{2}$ reducido). Sin embargo, la técnica del encerado no se ha implementado, hasta ahora, a gran escala en la agroindustria por los problemas impredecibles del desarrollo de frutas insípidas (Hagenmeier y Shaw, 1992) y de la maduración irregular (Smith et al., 1987). Encerando frutos de gulupa (Passiflora edulis Sims.), Montaño y Pachón (2006) encontraron que el encerado con Ceratec (emulsión a base de carnauba) no es un método viable para el almacenamiento, sino el empacado en la película plástica Vinilpel y la refrigeración a $6^{\circ} \mathrm{C}$ mostraron los mejores resultados.

Toivonen (2003) destaca que coberturas comestibles han mostrado modificar significativamente la atmósfera interna del fruto y controlar la pérdida de agua, sin embargo, hasta ahora es muy difícil diferenciar cual de los dos es el efecto más importante en la reducción del deterioro poscosecha de los productos.

Siendo una baja temperatura la posibilidad más importante de desacelerar el proceso de deterioro de las frutas frescas (Gallo, 1996); para una conservación óptima de la curuba recomiendan Landwehr y Torres (1995) 7 a $8^{\circ} \mathrm{C}$ y Gallo (1996) 6 a $8^{\circ} \mathrm{C}$. Téllez et al. (1999) observaron que frutos de curuba, almacenados a $8^{\circ} \mathrm{C}$, perdieron más lento su peso y consistencia, con una intensidad respiratoria más baja, comparado con el tratamiento a $20^{\circ} \mathrm{C}$. Como la curuba es un fruto que madura rápidamente bajo condiciones apropiadas, el trabajo tuvo como objetivo, evaluar las características fisicoquímicas y fisiológicas en poscosecha de dos cultivares almacenados a dos temperaturas de almacenamiento (ambiente y re- 
frigeración) y sometidos al encerado, con el fin de identificar un tratamiento adecuado, que permita retardar la maduración, garantizar una calidad sensorial y nutricional y extender el tiempo de la oferta de este fruto en el mercado nacional y la exportación.

\section{MATERIALES Y MÉTODOS}

Los frutos de curuba usados en este trabajo corresponden a dos cultivares mejorados y micropropagados in vitro, 'Ruizquin 1' y 'Ruizquin 2'; con resistencia a la antracnosis (Colletotrichum sp.) y tolerantes a la roña (Cladosporium sp.). Fueron seleccionados en una plantación ubicada en la vía Facatativá-Anolaima en Cundinamarca (2.400 msnm, $14,5^{\circ} \mathrm{C}$ de temperatura promedio y $2.500 \mathrm{~mm}$ de precipitación anual), rotulados aún en $100 \%$ de color verde, con sanidad total. Fueron cosechados a los 100 días de desarrollo aproximadamente a partir de la apertura de la flor, justamente, de acuerdo con Lizana et al. (1995), cuando la fruta tenía $75 \%$ de verde y $25 \%$ de amarillo (verde amarillo) para los estudios de fruta sin encerar y entre un $100 \%$ de verde (verde) y verde amarillo para los estudios de fruta encerada. Luego los frutos se transportaron en cajas plásticas a Bogotá y empacaron en cajas de cartón de 40x30x12 cm para su almacenamiento.

En el Laboratorio de Poscosecha de Frutas, Departamento de Ingeniería Civil y Agrícola, Universidad Nacional de Colombia, Bogotá, los frutos de ambos cultivares fueron almacenados en fresco sin encerarlos durante 25 días, y durante 55 días los que recibieron tratamiento con cera. Se usaron las temperaturas ambiente de $20 \pm 1^{\circ} \mathrm{C}$ (53\% humedad relativa [HR]) y de refrigeración en cuarto frío de $8 \pm 1^{\circ} \mathrm{C}$ ( $\left.85 \% \mathrm{HR}\right)$. La cera utilizada fue "Primafresh" (SC Johnson®), la cual fue aplicada sin tratamiento previo, el día mismo de su llegada al laboratorio, en forma manual, utilizando una esponja. Primafresh es una mezcla precisa de ésteres, ácidos-grasos-sacarosa, que al cubrir el fruto, forma una película inodora, incolora e insabora que restringe la difusión gaseosa y del vapor de agua a través de la cutícula (Vélez, 2003) y cuenta con la aprobación de la agencia Food and Drug Administration (FDA) de los Estados Unidos para usos en productos hortofrutícolas de exportación.

Se midió la intensidad respiratoria en cada uno de los cultivares, a intervalos de tres días, realizando tres repeticiones por temperatura utilizando un respirómetro que funciona mediante una trampa de hidróxido de potasio que atrapa $\mathrm{el}_{2}$ del ambiente, y una trampa de hidróxido de Bario empleando un tubo de Petenkoffer para capturar el $\mathrm{CO}_{2}$ expulsado por el fruto. Por la duración de este método no fue posible realizar la medición de los dos cultivares el mismo día, por tanto el 'Ruizquin 2' se evaluó con un día de diferencia con respecto al 'Ruizquin 1'. Se seleccionaron y marcaron al inicio de las mediciones, tres grupos o muestras de frutas por cultivar, temperatura y tratamiento, de manera que pesara cada uno aproximadamente $1 \mathrm{~kg}$, para medir la respiración en grupos homogéneos durante todo el estudio en la siguiente forma: (a) se tomó cada muestra y se introdujo en la cámara de respiración, se conectó la bomba de aire marca Pet Pro (Life 202), y se dejó pasar el aire ambiente únicamente, a través de la trampa de hidróxido de potasio $(0,1 \mathrm{~N})$ y la cámara de respiración, durante 15 segundos; (b) se tomaron $30 \mathrm{~mL}$ de hidróxido de Bario $(0,1$ N) y se introdujeron en el tubo de Petenkoffer, se conectó este con la manguera que venía de la cámara de respiración y se constató que por el tubo pasaran 13 burbujas de aire en 10 segundos, dejándose pasar el aire con el $\mathrm{CO}_{2}$ expulsado por la respiración de la fruta durante $20 \mathrm{~min}$; (c) se suspendió el flujo de aire desconectando la bomba y se tomaron $10 \mathrm{~mL}$ de hidróxido de bario $\left(\mathrm{Ba}(\mathrm{OH})_{2}\right)$ del tubo Petenkoffer, para titularlo doblemente con ácido oxálico $\left(\mathrm{C}_{2} \mathrm{H}_{2} \mathrm{O}_{4}\right), 0,1 \mathrm{~N}$, con el uso fenolftaleína, se realizaron al día tres blancos de $10 \mathrm{~mL}$ de $\mathrm{Ba}(\mathrm{OH})_{2}$ y se titularon con ácido oxálico $0,1 \mathrm{~N}$ en presencia de fenolftaleína; (d) los datos del volumen de ácido oxálico gastado en cada caso se registraron para el cálculo de 
la intensidad respiratoria utilizando la siguiente fórmula: $\mathrm{IR}=[(\mathrm{Vb}-\mathrm{Vm}) \mathrm{mL} \times \mathrm{N} \times 22 \mathrm{mg} \mathrm{CO}$ meq / ( $\mathrm{W} \times \mathrm{t})$ ], donde: $\mathrm{Vb}=$ Volumen $\mathrm{de}_{2} \mathrm{H}_{2} \mathrm{O}_{4}$ en $\mathrm{mL}$, gastado en titular el blanco, $\mathrm{Vm}=$ Volumen de $\mathrm{C}_{2} \mathrm{H}_{2} \mathrm{O}_{4}$ en $\mathrm{mL}$, gastado en titular la muestra, $\mathrm{N}$ $=$ Normalidad del $\mathrm{C}_{2} \mathrm{H}_{2} \mathrm{O}_{4} ; \mathrm{W}=$ Peso de la muestra en $\mathrm{kg}$; $\mathrm{t}=$ Tiempo en horas de flujo continuo de aire a través de sistema; (e) para graficar el comportamiento de la curuba tomada para el ensayo a lo largo del tiempo, se obtuvo un valor promedio diario de las tres repeticiones efectuadas y se trazaron las curvas de comportamiento.

Para el análisis fisicoquímico de la corteza se realizaron mediciones cada tercer día, con tres repeticiones por parámetro. La consistencia se midió con un penetrómetro marca Bertuzzi. Los sólidos solubles totales (SST) se midieron con un refractómetro, marca Kikuchi Trading (Japón). Para la determinación de la acidez total titulable se utilizó fenolftaleína $(0,1 \%)$ como indicadora y se tituló con $\mathrm{NaOH}(0,1 \mathrm{~N})$, este valor se expresó en porcentaje de ácido cítrico.

Para el análisis estadístico, el diseño utilizado corresponde a uno completamente al azar, con cuatro condiciones de almacenamiento, en un factorial $2 \times 2$, se emplearon un total de tres repeticiones por condición. Se aplicó el análisis de varianza multivariado y luego se realizó la prueba 'F' de significancia al 1\% y al $5 \%$ de probabilidad.

\section{RESULTADOS Y DISCUSIÓN}

\section{Intensidad respiratoria}

Los cultivares de curuba analizados se comportaron como climatéricos, lo cual coincide con los resultados en curuba de Lizana et al. (1991), Landwehr y Torres (1995), Urrutia (1995) y Gallo (1996). El climaterio, según Carmona (2001), es definido como un periodo de cambio en la tasa de respiración que acompaña al proceso de maduración de los "frutos climatéricos", mientras que en los "no climatéricos" la tasa de respira- ción no aumenta después de alcanzar el fruto su máximo crecimiento. Osterloh et al. (1996), afirman que el climaterio es consecuencia de los procesos de maduración y a causa del aumento en la energía para la maduración del fruto.

Para el cv. Ruizquin 1, encerado y a $20^{\circ} \mathrm{C}$, la respiración inició con un valor de $54,47 \mathrm{mg} \mathrm{CO}{ }_{2} \cdot \mathrm{kg}$ ${ }^{1} \cdot \mathrm{h}^{-1}$ el día de la recolección, para luego descender lentamente hasta un valor mínimo de $28,69 \mathrm{mg}$ $\mathrm{CO}_{2}$ en el día 21 , a continuación se inició un ascenso hasta su punto máximo $\left(54,66 \mathrm{mg} \mathrm{CO}_{2}\right)$ en el día 31, descendiendo luego hasta $40,94 \mathrm{mg} \mathrm{CO}_{2}$ en el día 34 (figura 1). El comportamiento mostrado por este cultivar a dichas condiciones de almacenamiento es típico de un fruto climatérico (Wills et al., 1998).

Para el mismo cultivar, a $20^{\circ} \mathrm{C}$, sin encerado, el valor del pico climatérico fue mayor, $77,31 \mathrm{mg}$ $\mathrm{CO}_{2} \cdot \mathrm{kg}^{-1} \cdot \mathrm{h}^{-1}$, en el día 11 obteniéndose en el día 25 daño total de la fruta (figura 1). Zhuang et al. (1997) afirman que temperaturas "súper-óptimas" de almacenamiento pueden resultar en una aceleración de la senescencia inducida oxidativamente.

La máxima intensidad respiratoria del cv. Ruizquin 1 a $20^{\circ} \mathrm{C}$ encerado, fue $29 \%$ menor que en frutos sin este tratamiento y postergó 20 días el periodo de aparición del climaterio. Las curubas bajo las condiciones de encerado a temperatura ambiente superaron el tiempo esperado de conservación, según lo afirmado por Landwehr y Torres (1995) que exista la posibilidad de almacenar la curuba a temperatura ambiente y HR de 90-95\%, por un tiempo de tres semanas (aprox. 24 días) utilizando tratamientos alternos como el encerado.

Para los frutos del cv. Ruizquin 1, encerados y a $8^{\circ} \mathrm{C}$, la curva inició con un valor de $65,48 \mathrm{mg}$ $\mathrm{CO}_{2} \cdot \mathrm{kg}^{-1} \cdot \mathrm{h}^{-1}$ en el día cero, para luego bajar paulatinamente hasta un valor mínimo de $14,52 \mathrm{mg}$ $\mathrm{CO}_{2}$ en el día 19, subiendo luego gradualmente hasta un valor máximo de $38,14 \mathrm{mg} \mathrm{CO}_{2}$, en el día 31, para descender más tarde hasta un valor de $16,52 \mathrm{mg} \mathrm{CO}$ en el día 45, incrementándose 


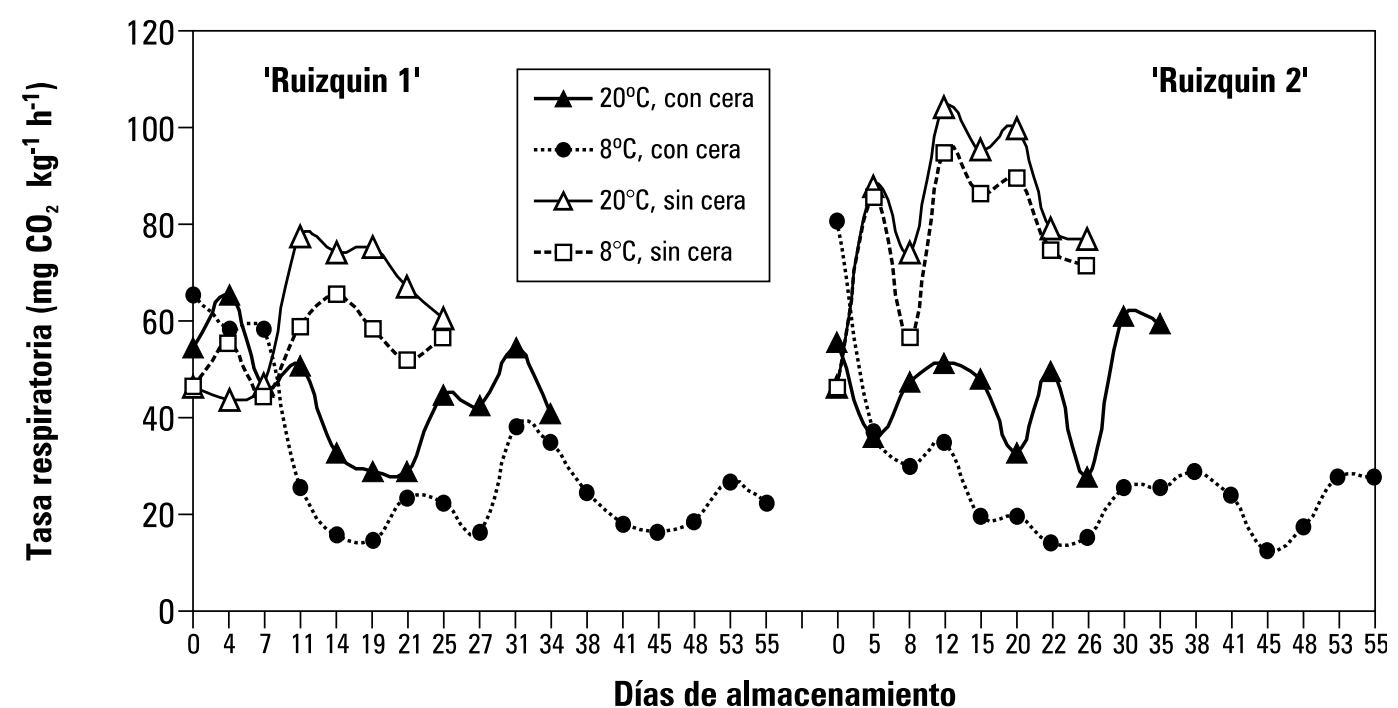

Días de almacenamiento

Figura 1. Intensidad respiratoria en frutos de curuba cvs. Ruizquin 1 (izquierda) y Ruizquin 2 (derecha), sin 0 con encerado, almacenados a temperatura ambiente $\left(20^{\circ} \mathrm{C}\right)$ o refrigerados a $8^{\circ} \mathrm{C}$.

luego hasta $26,71 \mathrm{mg} \mathrm{CO}_{2}$ (día 53) y 22,17 mg $\mathrm{CO}_{2}$ (día 55). Este último incremento se debe quizás al deterioro y caída de algunas capas de la cera sobre el fruto, debido a la manipulación y el ablandamiento del mismo.

Para los frutos refrigerados y sin cera del mismo cultivar, el valor máximo de la intensidad respiratoria fue de $65,41 \mathrm{mg} \mathrm{CO}_{2}$, en el día 14 . El tratamiento con cera en 'Ruizquin $1^{\prime}$ a $8^{\circ} \mathrm{C}$ hizo que la máxima intensidad respiratoria fuera un $42 \%$ menor que la de la fruta sin tratamiento previo y postergó el periodo de aparición del climaterio en 17 días. En el caso anterior la combinación de la aplicación de cera más una temperatura de refrigeración, bajó notablemente la intensidad respiratoria del fruto y prolongó su vida poscosecha (aplazamiento del pico respiratorio).

El cv. Ruizquin 1, con cera, sin importar la temperatura, presentó un mismo periodo de posible inicio de la senescencia, alrededor del día
31. Además reaccionó, independientemente de la temperatura, a la aplicación de cera como algunos autores afirmaron, en cuanto a ventajas como reducción de la intensidad respiratoria y alargamiento de la vida útil 15 días (en este estudio se postergó 20 días la aparición del pico climatérico), formando una barrera física adicional contra ataques de microoganismos y mohos, mencionado por Fonade (1988).

Para los frutos del cv. 'Ruizquin 2', encerada y a $20^{\circ} \mathrm{C}$, la respiración sigue un comportamiento oscilatorio, inicia con un valor de $55,7 \mathrm{mg} \mathrm{CO} \cdot \mathrm{kg}^{-1} \cdot \mathrm{h}^{-1}$, para luego bajar hasta un mínimo de $27,65 \mathrm{mg}$ $\mathrm{CO}_{2}$ en el día 26, a continuación elevarse hasta un máximo valor de $61,2 \mathrm{mg} \mathrm{CO}_{2}$ en el día 30 y luego descender (hasta 59,29 $\mathrm{mg} \mathrm{CO}$ ) en el día 35 (figura 1). En los frutos del mismo cultivar a igual temperatura pero sin encerar, la intensidad respiratoria llega a un valor máximo $(104,08$ $\mathrm{mg} \mathrm{CO}_{2}$ ) en el día 12. La aplicación de cera en los frutos de este cultivar, a temperatura ambiente, 
hizo disminuir considerablemente el valor del pico respiratorio en un $42 \%$ y retrasó su aparición 20 días, como lo indicado por Fonade (1988).

Para los frutos de 'Ruizquin 2', encerada y a $8^{\circ} \mathrm{C}$, se inició la intensidad respiratoria con un valor de $\left.80,92 \mathrm{mg} \mathrm{CO} \cdot \mathrm{kg}^{-1} \cdot \mathrm{h}^{-1}\right)$, en el día cero para luego bajar bruscamente (hasta $14,07 \mathrm{mg} \mathrm{CO}$ ), en el día 22 y posteriormente subir hasta $28,97 \mathrm{mg}$ $\mathrm{CO}_{2}$ en el día 38, y más tarde, descender hasta el día 45, continuando después un ascenso hasta $27,81 \mathrm{mg} \mathrm{CO}_{2}$ en el día 55. En este caso la curva respiratoria en el tiempo fue mucho más suave con un máximo mucho más bajo, que observando el mismo cultivar a igual temperatura, pero sin cera. Para el mismo cultivar, a $8^{\circ} \mathrm{C}$ y sin cera, la máxima intensidad respiratoria se presentó en el día $12\left(94,72 \mathrm{mg} \mathrm{CO}_{2}\right)$.

La mayor intensidad respiratoria de los dos cultivares encerados, se midió a los 31 y 30 días de almacenamiento a temperatura de $20^{\circ} \mathrm{C}$ y a los 31 y 38 días a temperatura de $8^{\circ} \mathrm{C}$ para 'Ruizquin 1' y 'Ruizquin 2' respectivamente. En frutos 'Ruizquin 2' se retrasó 7 días más el climaterio a $8^{\circ} \mathrm{C}$, al compararla con los 'Ruizquin 1 '.

Las tasas más bajas de respiración en frutos encerados fueron encontradas en curubas 'Ruizquin 2', mantenidos a $8^{\circ} \mathrm{C}$. La diferencia de reacción fisiológica observada entre cultivares también la encontraron Osterloh et al. (1996), los cuales afirman que la variación entre variedades de la misma especie puede llegar a ser de hasta 2,5 veces. El efecto del encerado sobre la intensidad respiratoria de los cultivares estudiados en este estudio fue mucho más marcada que el de la temperatura.

La tendencia general de las curvas tanto en frutas enceradas como en las no enceradas en cuanto a la consistencia del fruto fue decreciente (figura 2), tendencia similar a la reportada por Urrutia (1995) donde existió una disminución progresiva de la consistencia después de 28 días de almacenamiento, fenómeno ocasionado por pérdida de turgencia celular, entre otros.
Se encontró una menor tasa de ablandamiento al aplicar la cera y conservar los frutos a $8^{\circ} \mathrm{C}$ (figura 2). En este caso la aplicación de cera disminuyó considerablemente la pérdida de consistencia en ambos cultivares, siendo mayor esta en la fruta sin encerar. La disminución de turgencia es en gran parte resultado de la pérdida de agua por el fruto, procedente del jugo celular, mediante evaporación (Ramirez, 1991).

En los dos cultivares mantenidos a $8^{\circ} \mathrm{C}$ y encerados, la tasa de pérdida de consistencia se redujo con respecto a los frutos sin encerar en $0,26 \%$ en 'Ruizquin 1' y 1,2\% en 'Ruizquin 2'. El efecto más significativo de la aplicación de cera con respecto a una disminución del ablandamiento se dio en el cv. Ruizquin 2. La interacción del encerado con la temperatura de refrigeración sobre la pérdida de consistencia en los dos cultivares es significativo para R1 (al $P<0,05)$ y altamente significativo para R2 (al $P<0,01)$. El haber aplicado el encerado mantuvo la consistencia en los tejidos como reportan Leonardi et al. (2000), afirmando que un buen control del déficit de presión de vapor reduce la velocidad de transpiración y mantiene por mayor tiempo el agua en las células y la turgencia en los tejidos.

Se encontró una gran disminución de consistencia del fruto para los cvs. Ruizquin 1 y Ruizquin 2 encerados y a $20^{\circ} \mathrm{C}$, teniendo valores de $3,18 \%$ pérdida de firmeza por día para el primero y $3,7 \%$ para el segundo. A $20^{\circ} \mathrm{C}$ no tuvo lugar una reducción de pérdida de consistencia debida a la aplicación de cera en la superficie de los frutos de curuba. En el caso del cv. Ruizquin 1, encerado y mantenido a $20^{\circ} \mathrm{C}$, la pérdida de consistencia a los 31 días (aparición del climaterio) fue incluso del $98 \%$. Los resultados encontrados en este caso, confirman lo encontrado por Carmona (2001), solo si se combinan temperaturas seguras de almacenamiento y movimientos reducidos de aire junto con el empleo de ceras, se minimizan las pérdidas de agua. El efecto del encerado a temperatura ambiente pudiera haber sido distinto, quizás más positivo en la reducción de la 


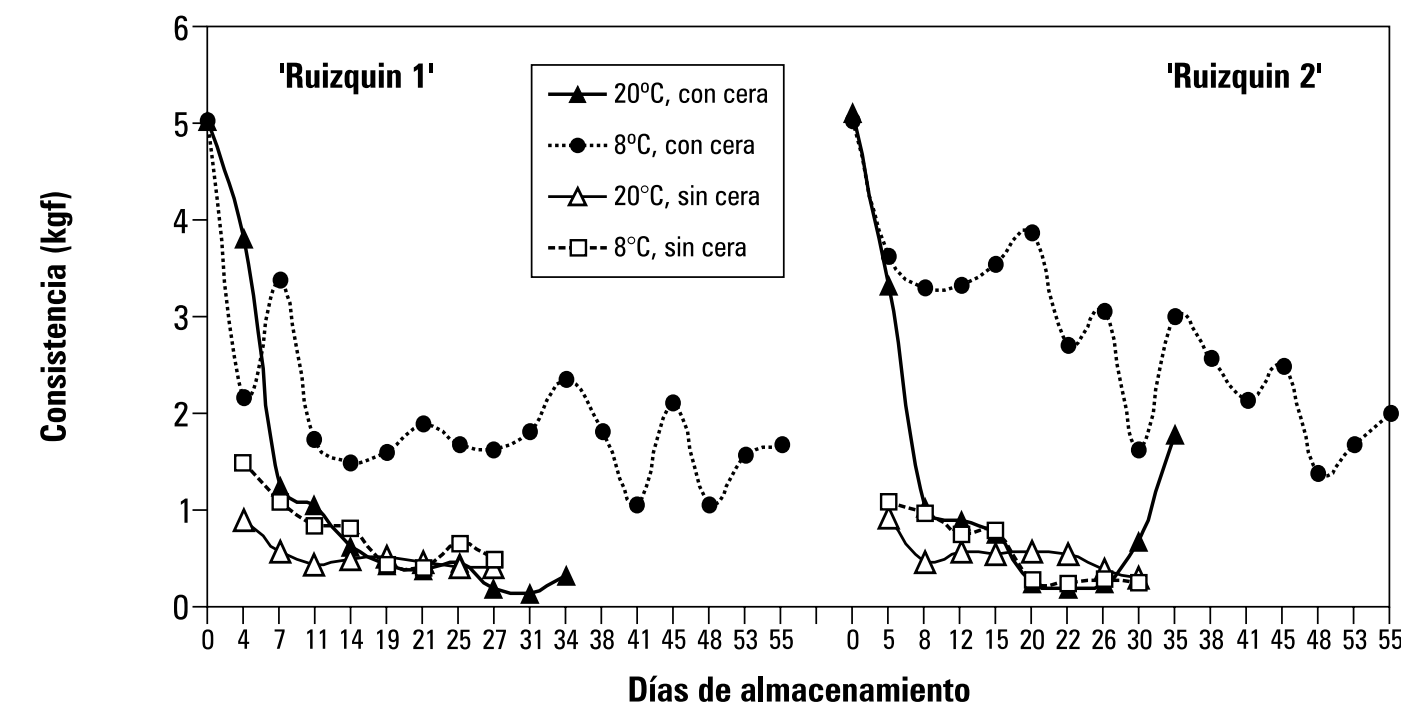

Figura 2. Consistencia en frutos de curuba cvs. Ruizquin 1 (izquierda) y Ruizquin 2 (derecha), con o sin encerado, almacenados a temperatura ambiente $\left(20^{\circ} \mathrm{C}\right)$ o refrigerados a $8^{\circ} \mathrm{C}$.

pérdida de consistencia del fruto, si se ensayara modificar la estructura de la cera empleada, pues como afirman Wills et al. (1998), la naturaleza de las superficies de recubrimiento es uno de los factores que aumenta la transpiración del producto vegetal, varios frutos poseen una $\mathrm{cu}$ bierta de cera casi impermeable al agua y vapor, restringiendo las pérdidas por evaporación del agua; la estructura del recubrimiento ceroso es más importante que su grosor, a mayor complejidad y ordenamiento en capas de la estructura, mayor resistencia a la transpiración.

Para el parámetro consistencia, el análisis de varianza indicó en ambos cultivares diferencia altamente significativa $(P<0,01)$ entre los tratamientos sin cera y con cera, aun mostrando un elevado coeficiente de variación (CV): 28,5 para 'Ruizquin 1' y 36,3 para 'Ruizquin 2', siendo este CV el mayor entre todos los parámetros medidos en este estudio. Aunque la consistencia de la pulpa está determinada genéticamente (Osterloh et al., 1996), el alto CV encontrado en este estu- dio, se puede expresar mediante las diferencias en las condiciones de crecimiento en campo, teniendo en cuenta que frutos desarrollados bajo alta radiación solar directa, forman cutículas más gruesas (Fischer, 1993) y por una posible desuniformidad del estado de madurez en que se cosecharon los frutos.

La menor pérdida de consistencia se encontró en 'Ruizquin 2', encerado y a $8^{\circ} \mathrm{C}$, donde a los 38 días (climaterio), el fruto aún conservaba más del $50 \%$ de su consistencia. Para el cv. Ruizquin 1 a los 31 días (climaterio), el fruto conservó el $36 \%$ de su turgencia habiendo perdido un $64 \%$.

$\mathrm{pH}$

El valor del pH para la curuba encerada fue mayor al de la sin cera (figura 3). En los dos cultivares el pH aumentó en poca magnitud con el tiempo, tendencia encontrado durante la maduración organoléptica de varios frutos climatéricos (Gortner et al., 1967). Los dos cultivares mostraron 


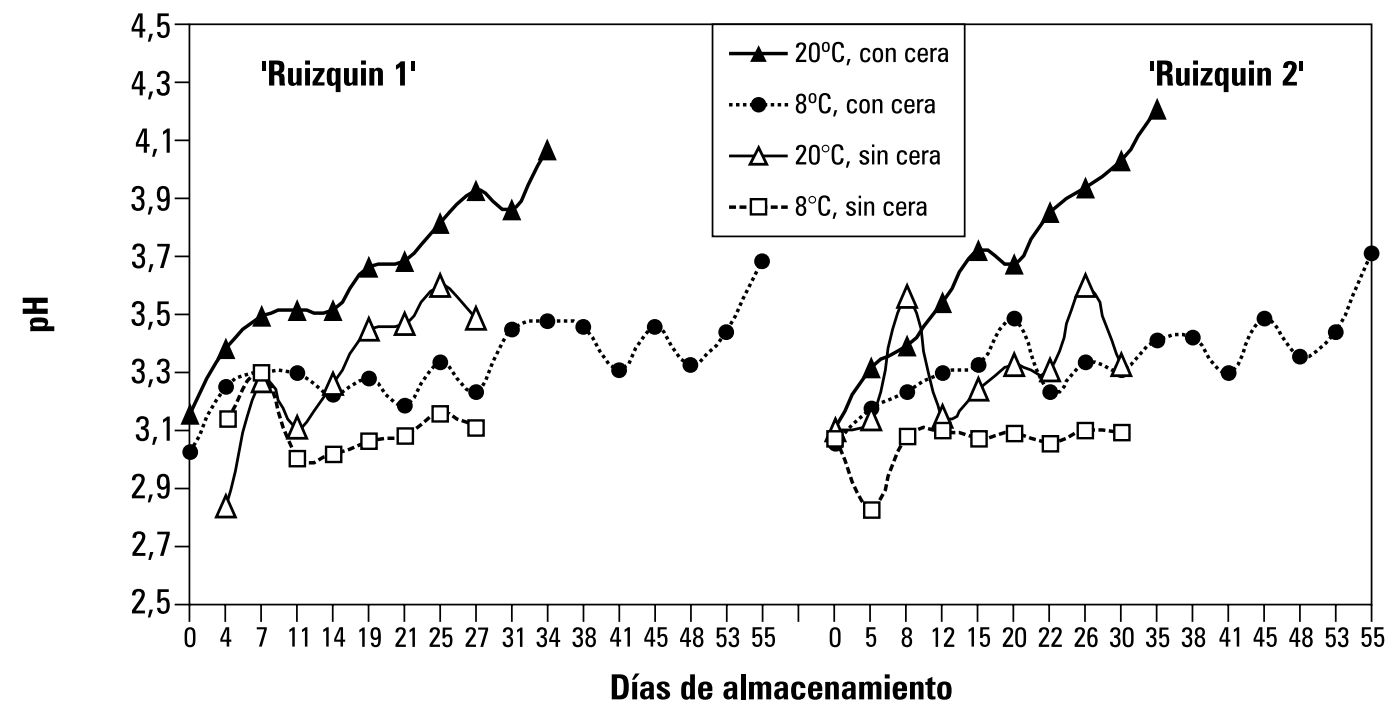

Figura 3. pH en frutos de curuba cvs. Ruizquin 1 (izquierda) y Ruizquin 2 (derecha), con 0 sin encerado, almacenados a temperatura ambiente $\left(20^{\circ} \mathrm{C}\right)$ o refrigerados a $8^{\circ} \mathrm{C}$.

un comportamiento muy similar. Sin mucha variación el pH aumentó ligeramente con el tiempo durante el almacenamiento, lo cual concuerda con los resultados de Villamizar (1991) y Urrutia (1995); este incremento, según Pantástico (1981), es debido a la reducción de la acidez. En frutos, más de $90 \%$ del volumen celular lo ocupa la vacuola, la cual, usualmente, es muy ácida, con pH inferior a 5 (Nanos y Kader, 1993); afirmación que coincide con los resultados encontrados con valores de $\mathrm{pH}$ en frutos encerados a $8^{\circ} \mathrm{C}$, de alrededor de 3,4 en los dos cultivares estudiados. Se observó que los frutos encerados tuvieron $\mathrm{pH}$ significativamente más altos $(P<0,01 \%)$ que los sin encerar, mostrando CVs entre 5,1 ('Ruizquin 1') y 5,3 ('Ruizquin 2').

\section{Sólidos Solubles Totales (SST)}

Los SST fueron en general bajos, a la mayor temperatura, sin importar el cultivar ni el encerado (figura 4). Con respecto al efecto del encerado, en general, las curvas muestran valores algo mayores cuando el fruto no está encerado, independientemente del cultivar. Estando el fruto encerado, al final del ensayo, a $20^{\circ} \mathrm{C}$, los SST de los dos cultivares fueron muy similares: 6,5 y $6,0^{\circ} \mathrm{Brix}$ al día 31 y 30 para 'Ruizquin 1' y 'Ruizquin 2', respectivamente. No se presentaron diferencias significativas entre cultivares.

En los dos cultivares encerados, a $8^{\circ} \mathrm{C}$, los SST más altos hacia el final del ensayo, los obtuvo 'Ruizquin 2' con $9,8^{\circ}$ Brix vs. solo $7,5^{\circ}$ Brix en 'Ruizquin 1', lo que podría indicar que en las curubas 'Ruizquin 2' tuvo lugar una más alta hidrólisis de almidón en azúcares, mejorando el sabor del fruto.

Los sólidos solubles no variaron mucho a lo largo del tiempo, este resultado se asemeja a las conclusiones de Benavidez y Rojas (1995), los cuales afirmaron que los ${ }^{\circ}$ Brix en la poscosecha de curuba oscilan entre 8 y 10. 


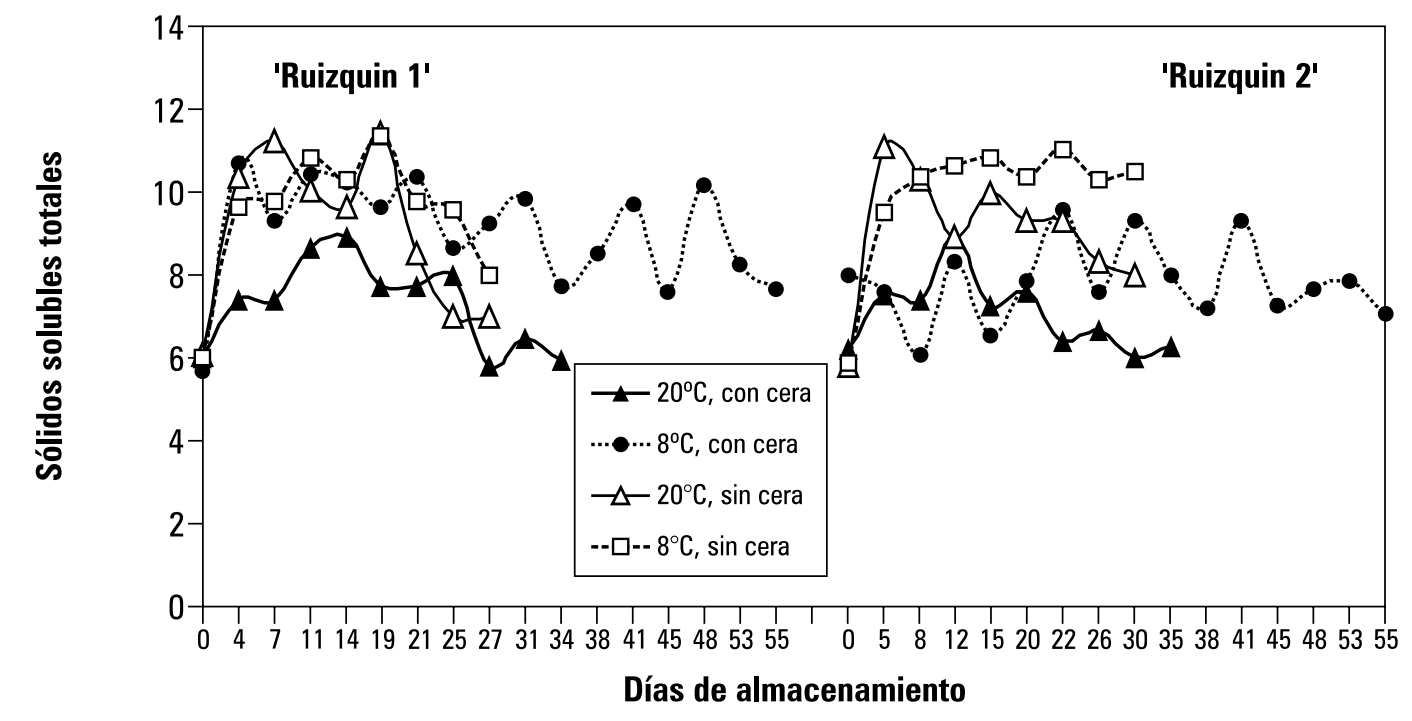

Figura 4. Contenido de sólidos solubles totales ( ${ }^{\circ}$ Brix) en frutos de curuba cvs. Ruizquin 1 (izquierda) y Ruizquin 2 (derecha), con o sin encerado, almacenados a temperatura ambiente $\left(20^{\circ} \mathrm{C}\right)$ o refrigerados a $8^{\circ} \mathrm{C}$.

\section{Acidez total titulable (ATT)}

La ATT para ambos cultivares disminuyó con el tiempo (figura 5); tendencia observada también por Lizana et al. (1991), Herrera (1991), Urrutia (1995) y Benavidez y Rojas (1995) en curuba. El hecho de dejar los frutos de curuba sin cera ocasionó una menor acumulación de ATT en los dos cultivares, debido, probablemente, por la mayor tasa de respiración que estos frutos exhibieron (figura 1) por lo cual se requiere una mayor cantidad de ácidos orgánicos como sustrato respiratorio que los cubiertos con cera.

Los ácidos orgánicos son, después de los carbohidratos, el depósito energético más importante para el fruto (Osterloh et al., 1996). Así, el presente estudio mostró que el encerado conserva mejor el porcentaje de ácido en las curubas y este efecto fue más significativo que la refrigeración.

El encerado tuvo un efecto altamente significativo $(P<0,01)$ sobre la ATT mostrando CVs entre
10,9 ('Ruizquin 1') y 10,7 ('Ruizquin 2'). No hubo diferencias significativas entre cultivares y entre fruta encerada, sin embargo el cv. Ruizquin 2 (con 2,1\%) a temperatura ambiente, para el mismo periodo de tiempo observado (31-32 días de almacenamiento), tendió a niveles menores que 'Ruizquin 1' (con 1,7\%).

\section{Relación Sólidos Solubles Totales / Acidez Total Titulable (SST/ATT)}

La figura 6 muestra que el comportamiento de la relación SST/ATT fue ligeramente creciente. En los dos cultivares, el no encerar aumentó la relación de SST/ATT, lo cual hace que su sabor sea algo más agradable que en frutos encerados, pues los azúcares son mayores que la acidez. La relación SST/ATT tiene suma importancia para el sabor del fruto y de su jugo, teniendo en cuenta que cuando el fruto tiene un contenido alto de azúcares, el nivel de los ácidos debe ser suficientemente elevado para satisfacer el gusto del consumidor (Osterloh et al., 1996). 


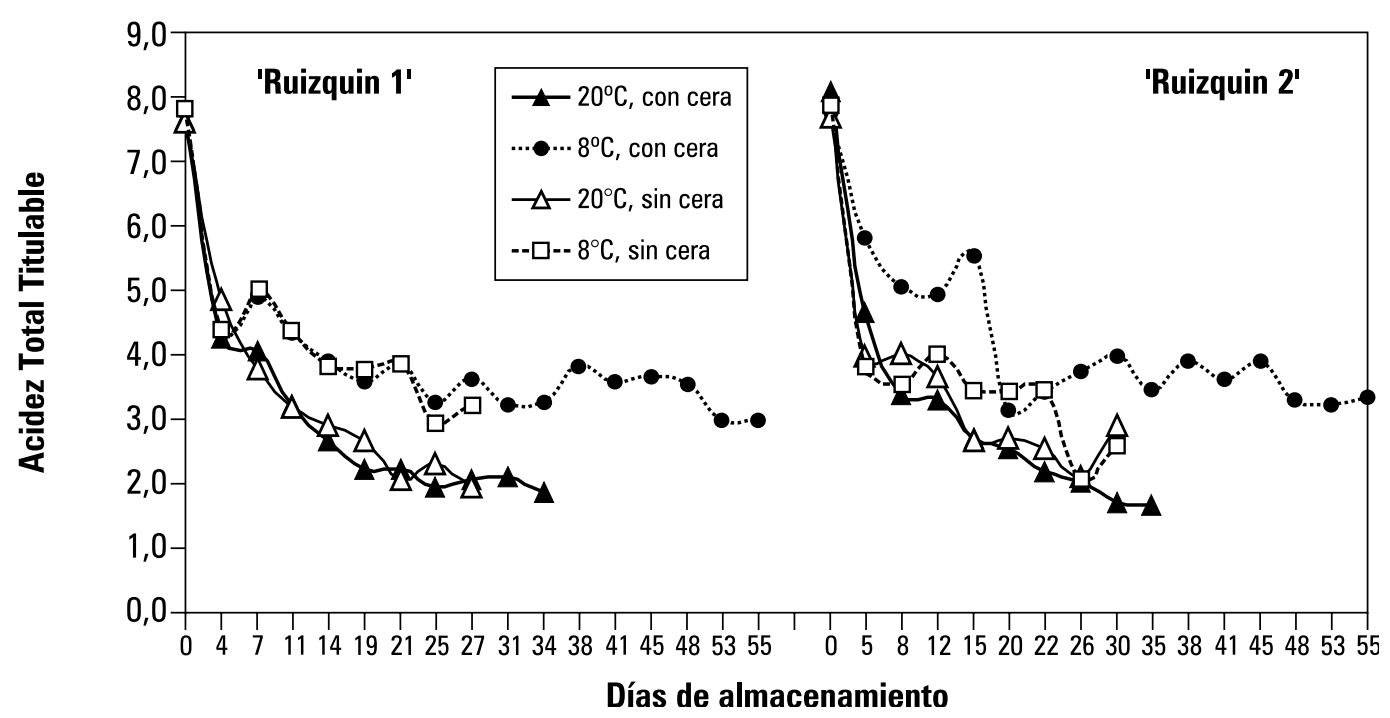

Figura 5. Variación de la Acidez Total Titulable (ATT) en frutos de curuba cvs. Ruizquin 1 (izquierda) y Ruizquin 2 (derecha), con o sin encerado, almacenados a temperatura ambiente $\left(20^{\circ} \mathrm{C}\right)$ o refrigerados a $8^{\circ} \mathrm{C}$.

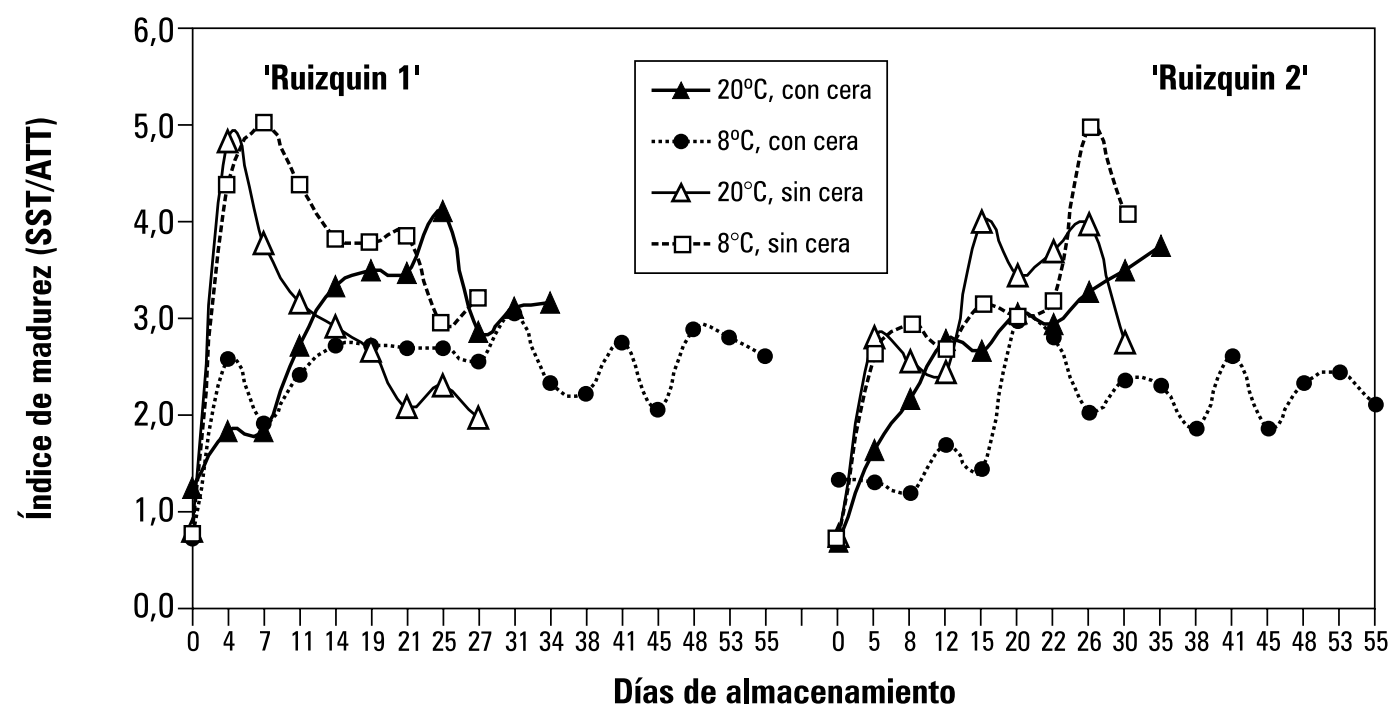

Figura 6. Relación Sólidos Solubles Totales / Acidez Total Titulable (SST/ATT) en frutos de curuba cvs. Ruizquin 1 (izquierda) y Ruizquin 2 (derecha), con o sin encerado, almacenados a temperatura ambiente $\left(20^{\circ} \mathrm{C}\right)$ 0 refrigerados a $8^{\circ} \mathrm{C}$. 
Para la relación SST/ATT, los análisis estadísticos indicaron que para los dos cultivares el efecto del encerado es altamente significativo $(P<0,01)$, los CVs fueron 18,8 ('Ruizquin 1') y 23,2 ('Ruizquin 2').

\section{Pérdida de peso}

Durante el almacenamiento en frutos con o sin encerado, la tendencia general fue la pérdida de peso con el transcurso del tiempo (figura 7); y, según Osterloh et al. (1996), la disminución de su peso es la suma de las pérdidas por transpiración y respiración; en frutos que respiran mucho se libera mayor cantidad de vapor de agua a la atmósfera de almacén.

Los frutos de curuba encerados perdieron menos peso que los frutos que están sin encerar, lo cual coincide con lo asegurado por Villamizar y Ospina (1995), que la cera reduce las pérdidas de agua (hasta un $50 \%$ durante el almacenamiento), la cual se traduce en pérdida de peso, y es más rápida e importante a temperaturas elevadas que a bajas, incluso con humedad relativa igual. También Valverde (1991) afirma que la cera Primafresh reduce el deterioro durante el almacenamiento, transporte y mercadeo por su acción contra la deshidratación al formar una película sobre el producto. Asimismo, Amarante y Banks (2001), reportan que el encerado reduce la permeabilidad al vapor de agua y el intercambio gaseoso entre el fruto y su entorno, ya que la cubierta externa bloquea los poros de la epidermis y se logra una reducción en la pérdida de agua de los tejidos retrasando la aparición de los síntomas por marchitamiento.

El cv. Ruizquin 1, encerado y almacenado a $8^{\circ} \mathrm{C}$, perdió más peso que el 'Ruizquin 2', por tanto, se puede concluir que éste último presenta una menor transpiración bajo estas condiciones. Las curubas enceradas y almacenadas a temperatura de $20^{\circ} \mathrm{C}$ no presentaron diferencias significativas entre cultivares.

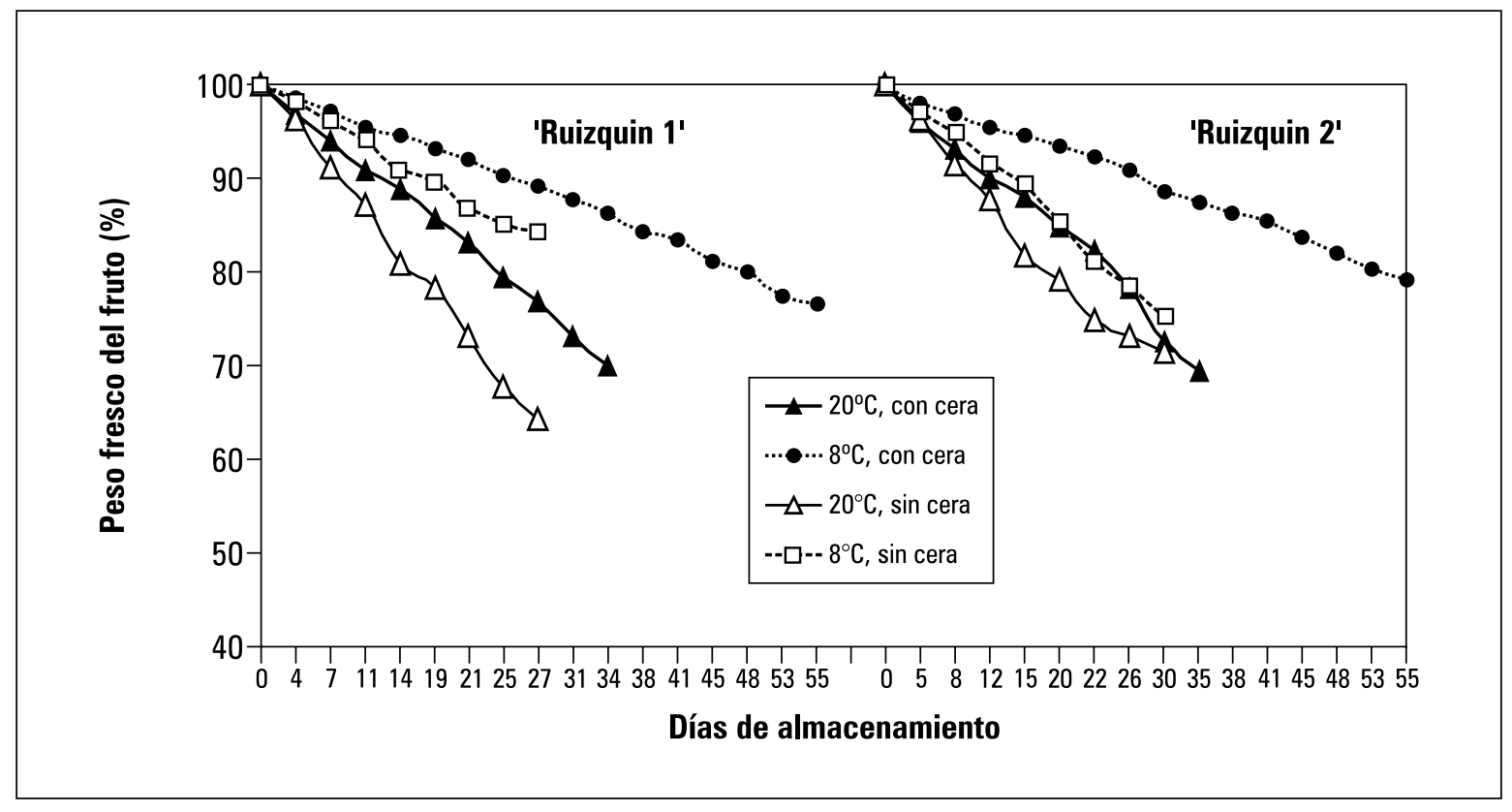

Figura 7. Pérdida de peso en frutos de curuba cvs. Ruizquin 1 (izquierda) y Ruizquin 2 (derecha), con o sin encerado, almacenados a temperatura ambiente $\left(20^{\circ} \mathrm{C}\right)$ o refrigerados a $8^{\circ} \mathrm{C}$. 
Para la pérdida de peso, el análisis de varianza indicó en ambos cultivares diferencia altamente significativa $(P<0,01)$ entre los tratamientos de encerado y no encerado, mostrando CVs entre 11,2 ('Ruizquin 1') y 16,1 ('Ruizquin 2').

\section{Coloración del fruto}

Las curubas de ambos cultivares a $8^{\circ} \mathrm{C}$ y enceradas, presentaron un tiempo máximo de almacenamiento en buenas condiciones de 30 días, aproximadamente, hasta alcanzar un color "amarillo puntas verdes" por lo cual se recomiendan estas condiciones para exportar y guardar durante largos periodos de tiempo por considerarse óptimas para conservar la consistencia, el peso inicial, bajar la tasa respiratoria y mantener un
pH adecuado. La curuba a $20^{\circ} \mathrm{C}$ y encerada se pudo almacenar durante un periodo de 21 a 25 días aproximadamente, alcanzando un color entre "amarillo puntas verdes y amarillo".

La curuba encerada, a los mismos días de almacenamiento y en iguales condiciones de temperatura y humedad relativa, cambió más lentamente el color de verde a amarillo y presentó menores síntomas de deterioro, como manchas pardas (textura áspera), deshidratación y hongos que la fruta sin encerado. Aplicando la cera Primafresh no se observó una decoloración de la pulpa, descrita por Pérez (1993) debido al incremento de la actividad de la polifenoloxidasa que también puede inducirse mediante estrés hídrico en precosecha de las plantas.

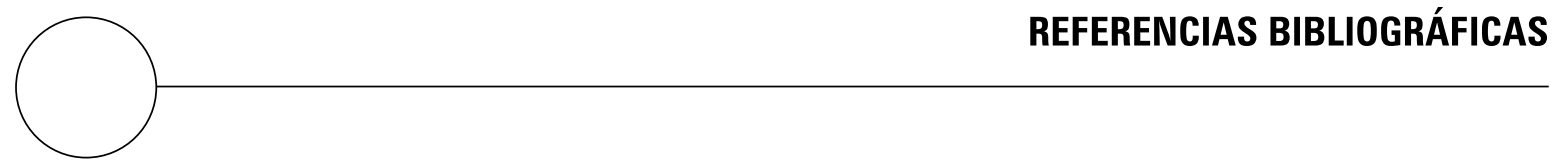

Amarante, C. y N.H. Banks. 2001. Postharvest physiology and quality of coated fruits and vegetables. Hort. Rev. 26, 238-261.

Angulo, R. y G. Fischer. 1999. Los frutales de clima frío en Colombia. La curuba. Ventana al Campo Andino 2(2), 24-28.

Baldwin, E.A.; M.O. Nísperos-Carriedo y R.A. Baker. 1995. Edible coatings for lightly processed fruits and vegetables. HortScience 30(1), 35-38.

Banks, N.H.; B.K. Dadzie y D.J. Cleland. 1993. Reducing gas exchange of fruits with surface coatings. Postharvest Biol. Technol. 3, 269-284.

Benavidez, J. y L. Rojas. 1995. Comparación del uso de envolturas plásticas y cera en la calidad de frutas y hortalizas frescas durante el almacenamiento. Trabajo de grado. Departamento de Ingeniería Agrícola, Facultad de Ingeniería, Universidad Nacional de Colombia, Bogotá.

Campos, T. 2001. La curuba: su cultivo. Instituto Interamericano de Cooperación para la Agricultura (IICA). Bogotá. 87 p.
Campos, T. 1992. El cultivo de la curuba (Passiflora mollissima H.B.K. Bailey) en Colombia. Acta Hort. 310, 215-231.

Carmona, G. 2001. Rol de la temperatura en el almacenamiento de productos frescos. Guía técnica postcosecha No 5. Consejo Nacional de Producción, Dirección de Calidad Agrícola, San José de Costa Rica. $17 \mathrm{p}$.

Fischer, G. 1993. Fisiología y manejo en pre y postcosecha. En: Frutales caducifolios. SIAC-Fenalce, Bogotá. pp. 97-117.

Fonade (Fondo Nacional de Desarrollo). 1988. Factibilidad para la producción y comercialización de la $\mathrm{Cu}$ ruba. Financiación Industrial Ltda., Bogotá. 220 p.

Gortner, W.A.; G.G. Dull y B.H. Krauss. 1967. Fruit development, maturation, ripening and senescence: a biochemical basis for horticultural terminology. HortScience 2, 141-144.

Gallo, F. 1996. Manual de fisiología, patología postcosecha y control de calidad de frutas y hortalizas. Convenio Sena-Reino Unido, Armenia. 
Hagenmeier, R.D. y P.E. Shaw. 1992. Gas permeability of fruit coating waxes. J. Amer. Soc. Hort. Sci. 117, 105-109.

Herrera, A. 1991. Determinación de los índices de cosecha en Curuba Passiflora mollissima. Convenio Sena - Universidad Nacional de Colombia, Bogotá.

Hoyos, J. 1989. Frutales en Venezuela. Sociedad de Ciencias Naturales La Salle. Monografía No. 36. Caracas. pp. 210-211.

Kader, A.A. (ed.). 2002. Postharvest technology of horticultural crops. University of California Agriculture and Natural Resources. Publication 3311. Third edition. Oakland, California. pp. 39-55, 67-79, 135 $145,279-285,385-398$.

Landwehr, T. y F. Torres. 1995. Manejo postcosecha de frutas. Instituto Universitario Juan de Castellanos, Tunja. 233 pp.

Leonardi, C.; S. Guichard y N. Bertin. 2000. High vapor pressure déficit influences growth, transpiration and quality of tomato fruits. Scientia Hort. 84, 285-296.

Lizana, A, J.; Luza, S. Espina y A. Vega. 1991. Caracterización de la maduración postcosecha de frutos de Curuba. En: Memorias del Simposio Internacional de Passifloras. Universidad Nacional de Colombia, Palmira. pp. 201-205.

Montaño, A. y Pachón, A. 2006. Efecto del empaque, encerado y temperatura sobre las características fisicoquímicas y organolépticas de la gulupa (Passiflora edulis f. edulis) en poscosecha. Trabajo de grado. Facultad de Agronomía, Universidad Nacional de Colombia, Bogotá.

Nakasone, H.Y. y R.E. Paull. 1998. Tropical fruits. CAB International, Wallingford, Inglaterra. pp. 17-44.

Nanos, G.D. y A.A. Kader. 1993. Low $\mathrm{O}_{2}$-induced changes in $\mathrm{pH}$ and energy charge in pear fruit tissue. Postharvest Biol. Technol. 3, 285-291.

Osterloh, A.; G. Ebert; W.-H. Held; H. Schulz y E. Urban. 1996. Lagerung con Obst und Südfrüchten. Verlag Eugen Ulmer, Stuttgart (Alemania). 253 p.

Pantástico, E.R. 1981. Fisiología de postrecolección. Vol. 1. Ed. Limusa S.A. México. pp. 812-815.

Pérez, A.L.M. 1993. Efecto de una cobertura de ceras naturales y un ester de sacarosa, sobre el comportamiento en almacenaje refrigerado de paltas (Persea americana Mill.) cv. Fuerte en distintos niveles de madurez. Facultad de Agronomía, Universidad Católica de Valparaíso, Quillota, Chile. 80 p.
Ramírez, A. 1991.Comportamiento de la curuba almacenada a diferentes condiciones. Proyecto Postcosecha, Convenio Sena-Universidad Nacional de Colombia. Bogotá. pp. 7-17.

Smith, S.; J. Geeson y J. Stow. 1987. Production of modified atmosphere in deciduous fruits by the use of films and coatings. HortScience 22, 772-776.

Tafur, R. 2006. Propuesta frutícola para Colombia y su impacto en la actividad económica nacional, regional y departamental. En: Memorias 1er Congreso Colombiano de Horticultura. Sociedad Colombiana de Ciencias Hortícolas, Unibiblos, Bogotá. pp. 47-66.

Téllez, C.P.; G. Fischer y O. Quintero. 1999. Comportamiento fisiológico y físico-químico en la poscosecha de curuba de Castilla (Passiflora mollissima Bailey) conservada en refrigeración y temperatura ambiente. Agron. Colomb.16(1-3), 13-18.

Toivonen, P.M.A. 2003. Postharvest treatments to control oxidative stress in fruits and vegetables. pp. 225246. En: Hodges. D.M. (ed.). Postharvest oxidative stress in horticultural crops. Food Products Press, New York, London. 266 p.

Urrutia, G. 1995. Estudio sobre diferentes estados de madurez de la curuba (Passiflora mollissima) y su incidencia en el mantenimiento postcosecha. Trabajo final. Especialización en Frutales de Clima Frío. Facultad de Ciencias Agropecuarias, UPTC, Tunja.

Valverde, G.E. 1991. Aplicación de ceras protectoras en los productos de la horticultura. SC Johnson de Centroamérica S.A., San José de Costa Rica. 12 p.

Vélez, M.S. 2003. Comunicación personal. SC Johnson, Bogotá.

Villamizar, F. 1991. Comportamiento fisiológico de la curuba almacenada a 3 temperaturas $\left(18^{\circ} \mathrm{C}, 10^{\circ} \mathrm{C} \mathrm{y}\right.$ $6^{\circ} \mathrm{C}$ ) desde la cosecha hasta la senescencia. Convenio Sena-Universidad Nacional de Colombia, Bogotá. pp. 11-17.

Villamizar, F. y J. Ospina. 1995. Manejo tecnológico postcosecha de frutas y hortalizas. Sena Publicaciones. Bogotá. 84 p.

Wills, R.; B. McGlasson; D. Graham y D. Joyce. 1998. Postharvest an introduction to the physiology and handling of fruit vegetables and ornamentals. $C A B$ International, New York. 262 p.

Zhuang, H.; D.F. Hildebrand y M.M. Barth. 1997. Senescence of broccoli buds is related to changes in lipid peroxidation. J. Agr. Food Chem. 43, 2585-2591. 